\title{
Surface Patterning for Enhanced Protein Stability - Insights From Molecular Simulations
}

Avishek Kumar, Deepshikha Ghosh, and Mithun Radhakrishna*

Department of Chemical Engineering, Indian Institute of Technology (IIT) Gandhinagar, Palaj, Gujarat 382355, India

E-mail: mithunr@iitgn.ac.in

\section{Supplementary Information}

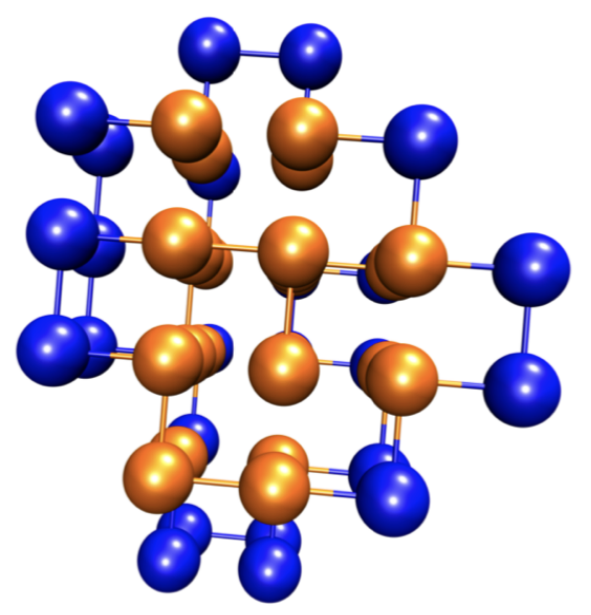

Figure S1: The ground (native/folded) state of the 48mer HP protein. The model protein is characterized by a hydrophobic core surrounded by a polar exterior. The ground state has an energy of $-34 \varepsilon_{\mathrm{HH}}$. Blue and Orange beads represent 'Polar' and 'Hydrophobic' groups respectively. There are $10 \mathrm{H}$ groups of the protein exposed in its folded state in an asymmetrical manner as shown here.
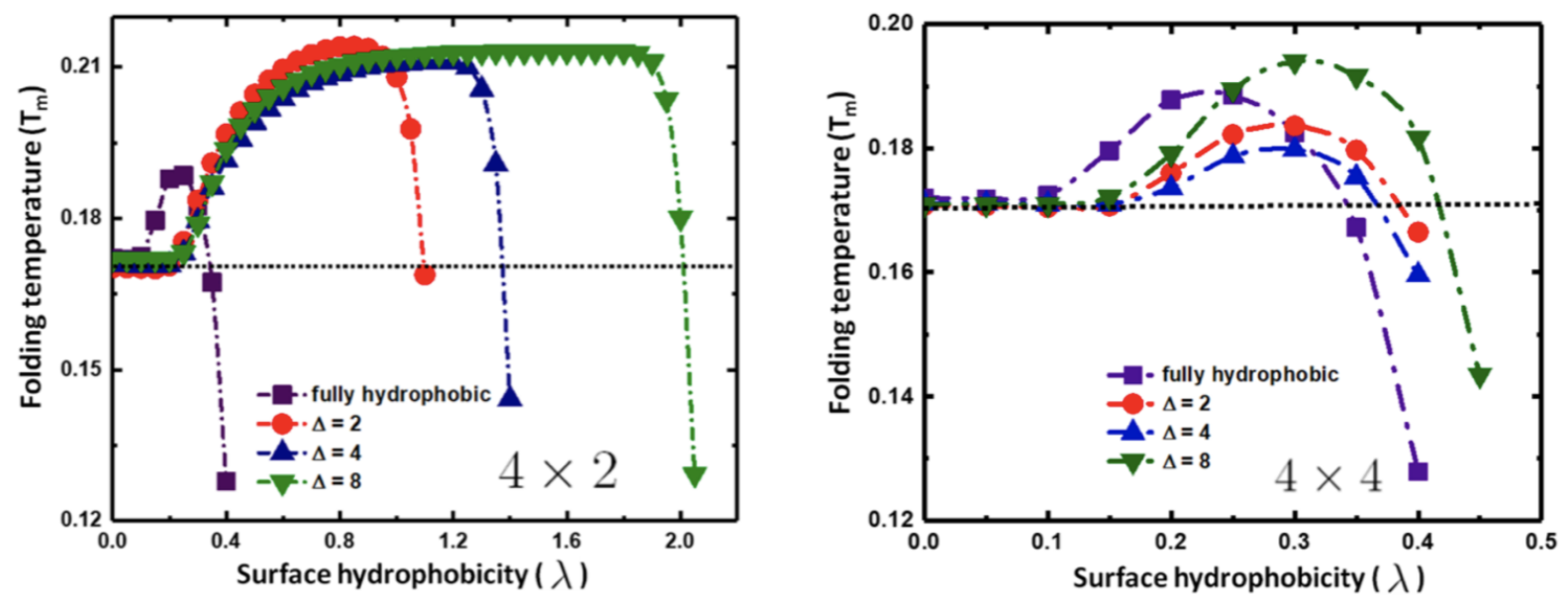
Figure S2: The effect of surface hydrophobicity on the folding temperature of $42 \mathrm{mer} \mathrm{HP}$ protein on different surface pattern arrangements. a) $4 \times 2$ and b) $4 \times 4$. The behavior of the protein on a homogeneous or a fully hydrophobic surface is also plotted for comparison. The thermal stability of the protein is greatly enhanced on $4 \times 2$ indicated by an increase in the folding temperature of the protein. The $4 \times 2$ patch is closest in complementarity to the exposed hydrophobic domain of the protein amongst the patterns studied. The protein is readily denatured on larger patches $(4 \times 4)$ due to protein-surface interactions 